\title{
Nepal Journal of Epidemiology
}

eISSN 2091-0800

Letter to the Editor

Open Access

\section{The relevance of digital mental healthcare during COVID-19: Need for innovations}

\author{
Sujita Kumar Kar ${ }^{1}$, Shailendra K Saxena ${ }^{2}$, Russell Kabir ${ }^{3}$
}

Correspondence: Dr Russell Kabir, School of Allied Health, Faculty of Health, Education, Medicine, and Social Care, Anglia Ruskin University, Chelmsford, United Kingdom.

Email: russell.kabir@aru.ac.uk

Received 28 Oct 2020/Revised 21 Dec 2020/Accepted 21 Dec 2020

Citation: Kar SK, Saxena SK, Kabir R. The relevance of digital mental healthcare during COVID-19: Need for innovations. Nepal J Epidemiol. 2020;10(4); 928-929.

DOI: $10.3126 /$ nje.v10i4.32519

This work is licensed under a Creative Commons Attribution 4.0 International License.

\section{Copyright @ 2020 CEA\& INEA. Published online by NepJOL-INASP.} www.nepjol.info/index.php/NJE

Sir,

The emergence of the COVID-19 pandemic and its rapid spread globally compromised healthcare delivery globally, including India. Digital health services are adopted worldwide to meet the healthcare needs of the people [1]. Digital platforms started providing online consultation for various medical conditions and mental illnesses [2]. As the digital platform is new for most patients and healthcare providers in India and ethical dilemmas were present with this modality of consultation, there was a reluctance to adopt it initially 3. However, the Government of India has issued guidelines for teleconsultation and promoted its use, which resulted in increasing acceptance of teleconsultation [1].

Tele-consultation for mental illnesses is running in India over the past two decades [4]. Diagnoses of most mental illnesses are based on detailed clinical history and interview of the patient (mental status examination). Physical examination, though an essential part of clinical evaluation, its role is less for making psychiatric diagnosis, unlike its essential role in various other medical disciplines. Hence, teleconsultation in mental health can suffice for the diagnosis of mental illnesses and psychotherapy and prescribing medications. In developing countries like India, the treatment gap for mental illnesses is approximately $85 \%$ [5]. Due to several reasons (social stigma, unawareness, financial crisis), people with mental illness cannot reach the mental health facilities for treatment.

COVID-19 may be considered an opportunity to enrol these deprived people in digital healthcare by creating awareness and providing digital healthcare facilities in remote areas by improving internet service. Teleconsultation can provide costeffective service at the doorstep with adequate client satisfaction [6] at the same time it facilitate early enrolment of patient in clinical care by reducing the duration of untreated illness [7]. Teleconsultations have been effectively used for various groups of patients (e.g: children \& adolescents, elderly, psychotherapy, addiction management) with diverse healthcare needs. Early evidences suggest that teleconsultation may serve as a cost-effective, convenient, feasible and effective modality to provide consultation to patient in the follow up care [8]. The healthcare workers working in primary care and wellness 
centres in remote areas may play a pivotal role in bridging the gap between patients with mental healthcare needs and mental health professionals. A parallel tele-networking involving the pharmacy may facilitate the delivery of medicines at doorstep following teleconsultation. Teleconsultation can facilitate multidisciplinary collaboration more easily than direct consultations in a time convenient manner. Use of digital educational materials and videos can pass on proper health education to a larger audience by reducing the task of treating clinician. Teleconsultation may be going to become more sensitive tool for assessment, diagnosis, communication, delivery of personalized care by using the concepts of artificial intelligence [9]. Researchers are using various algorithms to analyse data, which may help in accurate prediction related to diagnosis, selection of treatment as well as anticipating outcome.

Dr. Sujita Kumar Kar, Department of Psychiatry, King George's Medical University, Lucknow 226003, India.

Dr. Shailendra K Saxena, Centre for Advanced Research (CFAR), Faculty of Medicine; King George's Medical University Lucknow 226003, India.

Dr. Russell Kabir, School of Allied Health, Faculty of Health, Education, Medicine, and Social Care, Anglia Ruskin University, Chelmsford, United Kingdom.

Dated the 28 October 2020

\section{Acknowledgement}

None

\section{Competing interests}

There is no conflict of interest for any author of this manuscript. Authors' affiliations:

${ }^{1}$ Department of Psychiatry, King George's Medical University, Lucknow, India.

${ }^{2}$ Centre for Advanced Research (CFAR), Faculty of Medicine; King George's Medical University, Lucknow, India.

${ }^{3}$ School of Allied Health, Faculty of Health, Education, Medicine, and Social Care, Anglia Ruskin University, Chelmsford, United Kingdom.

\section{References}

1. Agrawal A. Bridging digital health divides. Science 2020; 369: 1050-1052.

https://doi.org/10.1126/science.abc9295

PMid:32855322

2. Romanick-Schmiedl S, Raghu G. Telemedicine - maintaining quality during times of transition. Nature Reviews Disease Primers 2020; 6: 1-2.

https://doi.org/10.1038/s41572-020-0185-X

PMid:32483168 PMCid:PMC7262488
3. Sousa A, Karia S. Telepsychiatry during COVID-19: Some clinical, public health, and ethical dilemmas. Indian Journal of Public Health 2020; 64: 245-246.

https://doi.org/10.4103/ijph.IJPH_511_20

PMid:32496267

4. Thara R, Sujit J. Mobile telepsychiatry in India. World Psychiatry 2013; 12: 84 .

https://doi.org/10.1002/wps.20025

PMid:23471809 PMCid:PMC3619179

5. Gautham MS, Gururaj G, Varghese M, et al. The National Mental Health Survey of India (2016): Prevalence, sociodemographic correlates and treatment gap of mental morbidity. Int J Soc Psychiatry. 2020 Jun;66(4):361-372.

https://doi:10.1177/0020764020907941

PMID: 32126902.

6. Marcin JP, Shaikh U, Steinhorn RH. Addressing health disparities in rural communities using telehealth. Pediatric Research 2016; 79: 169-176.

https://doi.org/10.1038/pr.2015.192 PMid:26466080

7. Ventriglio A, Torales J, Castaldelli-Maia J. Telepsychiatry and social psychiatry. Int J Soc Psychiatry 2017; 63: 387-388.

https://doi.org/10.1177/0020764017691552

PMid:28701084

8. Das S, Manjunatha N, Kumar CN, et al. Tele-psychiatric after care clinic for the continuity of care: A pilot study from an academic hospital. Asian Journal of Psychiatry 2020; 48: 101886.

https://doi.org/10.1016/j.ajp.2019.101886

PMid:31835142

9. Kuziemsky C, Maeder AJ, John O, et al. Role of Artificial Intelligence within the Telehealth Domain. Yearb Med Inform 2019; 28: 35-40.

https://doi.org/10.1055/s-0039-1677897

PMid:31022750 PMCid:PMC6697552 